Analogical Thinking in Ecology: Looking beyond Disciplinary Boundaries

Author(s): Mark Colyvan and Lev R. Ginzburg

Source: The Quarterly Review of Biology, Vol. 85, No. 2 (June 2010), pp. 171-182

Published by: The University of Chicago Press

Stable URL: http://www.jstor.org/stable/10.1086/652321

Accessed: 13/01/2011 01:24

Your use of the JSTOR archive indicates your acceptance of JSTOR's Terms and Conditions of Use, available at http://www.jstor.org/page/info/about/policies/terms.jsp. JSTOR's Terms and Conditions of Use provides, in part, that unless you have obtained prior permission, you may not download an entire issue of a journal or multiple copies of articles, and you may use content in the JSTOR archive only for your personal, non-commercial use.

Please contact the publisher regarding any further use of this work. Publisher contact information may be obtained at http://www.jstor.org/action/showPublisher?publisherCode=ucpress.

Each copy of any part of a JSTOR transmission must contain the same copyright notice that appears on the screen or printed page of such transmission.

JSTOR is a not-for-profit service that helps scholars, researchers, and students discover, use, and build upon a wide range of content in a trusted digital archive. We use information technology and tools to increase productivity and facilitate new forms of scholarship. For more information about JSTOR, please contact support@ jstor.org. 


\title{
ANALOGICAL THINKING IN ECOLOGY: LOOKING BEYOND DISCIPLINARY BOUNDARIES
}

\author{
Mark Colyvan \\ Sydney Centre for the Foundations of Science, University of Sydney, Sydney, New South Wales, \\ 2006, Australia \\ E-MAIL: MCOLYVAN@USYD.EDU.AU \\ Lev R. Ginzburg \\ Department of Ecology and Evolution, Stony Brook University, Stony Brook, NY 11794, USA \\ E-MAIL: LEV@RAMAS.COM
}

KEYWORDS

analogy, maternal effect, ratio dependence, allometries, philosophy of ecology, population ecology

\begin{abstract}
We consider several ways in which a good understanding of modern techniques and principles in physics can elucidate ecology, and we focus on analogical reasoning between these two branches of science. Analogical reasoning requires an understanding of both sciences and an appreciation of the similarities and points of contact between the two. In the current ecological literature on the relationship between ecology and physics, there has been some misunderstanding about the nature of modern physics and its methods. Physics is seen as being much cleaner and tidier than ecology. When compared to this idealized, fictional version of physics, ecology looks very different, and the prospect of ecology and physics learning from one another is questionable. We argue that physics, once properly understood, is more like ecology than ecologists have thus far appreciated. Physicists and ecologists can and do learn from each other, and, in this paper, we outline how analogical reasoning can facilitate such exchanges.
\end{abstract}

\section{INTRODUCTION}

$\mathrm{E}$ COLOGY AND PHYSICS have different subject matters and, on the face of it at least, quite different methods. But still there are similarities; for example, they both employ similar mathematical methods. There has been a lively ongoing discussion about the similarities and differ- ences between ecology and physics (e.g., Quenette and Gerard 1993; Lawton 1999; Turchin 2001; Berryman 2003; Lange 2005; O'Hara 2005), and this discussion has revolved around two main themes: whether ecology has laws like those of physics, and whether it is fruitful to employ some of the methods of physics in ecology. Although 
these issues are both of considerable interest and are closely related, we will focus mainly on the second, more general issue. We argue for a specific sense in which ecology can be advanced by looking towards physics, and we show that analogical reasoning between different branches of science is a very fruitful means of generating new hypotheses. In particular, we argue that advances have been made-and will continue to be made-in ecology as a result of keeping watchful eyes on ideas from physics and emulating some of the more successful strategies. Sometimes this involves exploring the idea that ecology has laws not unlike the laws of physics, and sometimes it involves thinking about the appropriate number of dimensions for the formulation of theories, as is common in physics.

First, we discuss some of the reasons advanced in support of the view that physics and ecology are different. After all, if ecology and physics are radically different, then it may be fruitless for ecologists to even try to learn from physics. However, we will argue that ecology and physics are not so different. The alleged dissimilarities between the two have been unintentionally exaggerated by some participants in the aforementioned debate, and this exaggeration seems to be a result of holding an idealized, unrealistic picture of how physics operates. With the misconceptions about physics cleared away, the differences between ecology and physics are much less significant.

We then discuss three examples of where ecology has made advances or where advances might occur as a direct result of the kind of analogical reasoning that we advocate. We finish by showing that this is not "physics envy" on our part; we believe that the use of analogical reasoning across different branches in science is generally very useful and is already widely used in physics. We also give a couple of examples where physics has learned from ecology and evolution via analogical reasoning. Throughout the discussion, we argue for similarities between ecology and physics, but we do this in order to show why these similarities matter: both ecology and physics are advanced by appreciating their similarities and availing them- selves of analogical reasoning to make the relevant connections.

\section{Ecology And Physics Are Not So DiffERENT}

Dale Lockwood's (2008) recent paper, "When logic fails ecology," is a clear and carefully articulated example of the case for the dissimilarity of physics and ecology. We should make it clear that Lockwood is not addressing the specific issue of analogical reasoning that we are interested in. Rather, his primary focus is on arguing against the claim that there are laws in ecology, but, in attempting to do this, he touches on several important points about the alleged differences between physics and ecology, and we suspect that he speaks for many on these issues. In any case, his paper advances a forceful case that needs addressing and provides a useful point of departure for our discussion in this section.

Lockwood starts by suggesting that he will set aside the issue of whether there are laws in physics and focus on whether there are laws in ecology. But as his own discussion demonstrates, and as we argued previously (Colyvan and Ginzburg 2003b), this is hard to do. The problem is that the best examples of laws are in physics, so one can either use those laws to serve as the basis for a definition of "law" and see whether the alleged laws of ecology measure up to that definition, or, alternatively, one can directly compare the alleged laws of physics with their counterparts in ecology. Given the extreme difficulty in providing an adequate definition of "law," we opted for the latter strategy (Colyvan and Ginzburg 2003b; Ginzburg and Colyvan 2004), concluding that either ecology does have some good candidates for laws, or that there are no laws in physics either (Colyvan and Ginzburg 2003b). Lockwood $(2007,2008)$ is a little bolder than we were and opts for the strategy of offering a definition (or at least a working definition) of a law as "a factual truth that is spatiotemporally universal, supports counterfactuals, and has a high level of necessity" (2008:58).

As definitions of "law" go, Lockwood's is not a bad one, but it would seem to rule 
out the existence of laws in physics. Although Lockwood says that he wishes to leave the issue of laws in physics aside, he adopts a definition of law so stringent that it rules out most of the usual candidates for laws anywhere. He is thus left advancing a general eliminitivist position about laws, according to which there are none. This was one of the options we left open, but we find it a rather unattractive option. Rather than set the bar so high for lawhood that there are none, it is better to be less stringent about what counts as a law-hence our countenance of laws with exceptions, laws with ceteris paribis clauses, and the like. Indeed, given that Lockwood goes on to argue for the differences between physics and ecology, we take it that he does not in fact mean to be advancing such a general elimination of laws. He does, it seems, believe that there are laws in physics and that his definition adequately captures them. We believe he is mistaken about the adequacy of his definition of "law," but the reasons for his mistake are both interesting and informative.

Lockwood takes laws of physics to be temporally universal: they are supposed to hold for all time. But, in physics, it is routinely suggested that the current laws did not hold during the very early period of the universe. We must take partial responsibility for this mistake, for we (and others) have focused on very simple cases from physics - namely, Newton's laws-for the purpose of ease of exposition. Perhaps there was a time when Newton's laws were thought to be universal in this sense, but modern laws in physics - if there are anyare generally no longer thought of in this way. Lockwood's mistake is to generalize from a limited number of simple cases from physics; in effect, he takes physics to be cleaner and simpler than it really is. Ecologists, more than anybody, are aware of the complexities and difficulties in ecology. It is not surprising then that when you consider a realistic and accurate picture of ecology, as Lockwood so admirably paints, and you compare this with an idealized, high school-level cartoon of physics, ecology comes out looking messier. However, as it turns out, almost all the claims that Lockwood makes about the failings of the candidate laws in ecology straightforwardly carry over to physics, once physics is properly understood. Lockwood points out the lack of additivity in ecology without realizing that this is also the case in physics (e.g., velocities in special relativity do not add). He also notes that in ecology there is a choice about how to represent the dynamics, but, again, this is the same in physics. There are different ways to represent quantum mechanics (e.g., Dirac and Hilbert's respective methods), as well as several different interpretations of the formalism (Hughes 1989). Even in classical Newtonian mechanics there are Lagrangian and Hamiltonian formulations (see Lyon and Colyvan 2008 for the significance of these different formulations). Lockwood notes that there is underdetermination of theory by data in ecology, but fails to appreciate that this is so in physics as well (see Duhem 1954, Lakatos 1970, and Quine 1980). There are also a number of contentious points in Lockwood's paper, such as non-standard views about logic, logical possibility, and what the counterfactual dependency requirement for laws involves, but these points do not bear directly on our purposes here, so we set them aside. There are, however, two claims that Lockwood makes that deserve closer inspection.

Lockwood suggests that laws in physics are exceptionless and discusses our example of a snowflake and a rock falling with different accelerations, contrary to Galileo's law that acceleration is independent of mass. Lockwood is quite right to point out that Galileo's law can be saved by positing differential friction from air resistance in these two cases. Moreover, he is correct that this is the standard account of how to understand such apparent violations of the law in question. Lockwood fails, however, to realize that such a move can always be made, and indeed can be made in ecology as well. For instance, whenever we see a population that is not growing according to the logistic equation, there is nothing to stop us from positing some complicating factor that saves the logistic equation. While it might be tempting 
to suggest that the difference between Galileo's law and the logistic equation is to be found in the empirical testability of the posited complicating factors, this will not work. The empirical demonstration of the complicating factors involved in the apparent failure of the logistic equation is straight forward-it is seen in the failure of the population to grow according to the logistic equation. This might seem ad hoc and even circular, but it is no more circular or ad hoc than similar cases in physics (e.g., conservation laws). It is very common to count a deviation from a relevant law as an empirical demonstration of some effect.

On a related point, Lockwood claims that there is a difference between physics and ecology in that idealizations required to get the laws to work in ecology are "unrealistic." But the idealizations in ecology are no more unrealistic than the idealizations in physics: point masses, inertial reference frames, incompressible fluids, and the like. As we suggested in our earlier paper (Colyvan and Ginzburg 2003b), the appropriate way to understand laws like Newton's first law (i.e., all bodies move with uniform motion unless acted upon by a force) is not in terms of describing any actual system. Maybe Lockwood is right and there are some systems with zero net force acting upon them, although it is not clear that his examples of books sitting on a shelf on a planet rotating on its axis and orbiting a star, which is, in turn, rotating around the galactic centre, are the best examples for his purposes. In any case, the laws of physics are not merely about such special cases, if indeed there are any; Newton's first law is supposed to inform us about other systems as well. Laws provide background conditions from which departures need to be explained (Ginzburg and Colyvan 2004). Think of Newton's first law as a complicated counterfactual about what would happen if a system were isolated from all forces, or as a description of an idealized system that has some similarity to the system under consideration. Once the role of such laws in physics are appreciated, we see that Lockwood's remarks about the candidate laws in ecology being about ideal popula- tions and thus unrealistic is exactly right, but, contrary to what he goes on to conclude, this is precisely why these are good candidates for laws. According to the view of laws that we advocate, invoking unrealistic, ideal setups is precisely what we should expect from a law. Most importantly, for our present purposes, physics and ecology are alike in this regard.

\section{Analogical Insights from Physics}

For all that has been said so far, there is no reason to believe that physics and ecology are so different that one cannot learn from the other. In particular, we have argued that there is no reason to take physics to be law-governed and ecology not to be law-governed. With this much of a connection in place, we want to proceed with establishing the usefulness of exchanging or borrowing methods and insights across these two disciplines. The historical question of how often such exchanges have occurred is an interesting one. Although there is evidence for at least some exchange of methods, we do not need to establish historical precedence in order to make our case, as our claim is about its usefulness. In order to demonstrate this, we also do not need to establish that there has been borrowing on a regular basis and that it has mostly been useful. Although that would suffice for our case, if true, it would likely be old news. Alternatively, we could argue directly for the usefulness of the kind of analogical reasoning we have in mind. This can be done by showing that the kinds of analogies in question could plausibly lead to advances in the relevant theories. We will employ both strategies, giving some historical evidence that analogical thinking has been used to good effect, but also offering other cases where it is at least plausible that analogical reasoning has been, or might have been, employed to good effect.

We now turn to the details and specific examples of how ecology can learn from physics. We will show how adopting some of the mathematical techniques employed in physics, by way of analogy, can be useful in ecology. 
TIME AS THE FOURTH DIMENSION IN METABOLIC ECOLOGY

One example demonstrating the potential of analogical thinking in ecology is the consideration of the dimension of the biological organism. The issue arises out of metabolic ecology-the field where metabolic rates are studied as a function of body size. Not only metabolic rates but also rates of reproduction, the longevity of organisms, and even their geographic density relate to body size in interesting ways (the best recent reference to the subject is a review by Brown et al. [2004] and a popular book by Whitefield [2006]). A longstanding problem in this area is how to explain why metabolic rate scales as $3 / 4$ the power of body size rather than $2 / 3$. Originally, it was suggested (Rubner 1883) that metabolism, as a property of surfaces, is quadratic with a linear dimension, whereas body size (weight) is cubic, so that $2 / 3$ is the expected slope of the line when metabolism is plotted against body weight (in log scales). Klieber (1932) had discovered, however, that in the interspecies comparison, $3 / 4$ is a better approximation.

The suggestion that a fourth dimension of some sort is involved in explaining the $3 / 4$ power is over 30 years old (Blum 1977). Hainsworth (1981), in his well-known book on physiology, briefly suggested that time may fill that role, and this picture clarified recently with the suggestion by Ginzburg and Damuth (2008) that generation time may well be the missing fourth dimension, to be added to the three spatial dimensions. According to this view, $3 / 4$ is the expected power in cross-species comparisons where there are great differences in generation times. The power in question is predicted to be $2 / 3$ within a single species, where sizes differ but longevity does not. The power can even be $1 / 2$ if, in addition to placing constraints on the time dimension (e.g., by fixing generation time), one also constrains one of the spatial dimensions (e.g., by comparing only humans of a particular height).

We will not bother with the details here, as they are not important for our present purposes. Our point is simply that the insight from physics of seeing time as a fourth dimension and thinking of objects as four-dimensional rather than as threedimensional can-and has-served as a springboard from which to consider generation time as a natural and inseparable dimension of an organism. The idea of linking space and time and thinking of them as different aspects of one thing (i.e., the space-time manifold) was developed to great advantage in physics over 100 years ago. The move to space-time as the basic framework in physics served as inspiration for investigating the analogous idea in ecology (Ginzburg and Damuth 2008). The idea is that, in physics, some phenomenon (e.g., the Lorentz contraction) is puzzling when considered in a three dimensional framework, but it is exactly what one would expect when the phenomenon in question is considered in a four-dimensional spacetime framework. The central idea of Ginzburg and Damuth (2008) was to explore this idea as a way of approaching the puzzling $3 / 4$ power appearing in metabolic ecology.

But even without this historical case, it should be clear that thinking about organisms in this four-dimensional way might have been arrived at via analogical reasoning from relativity theory. Recall that we are arguing that such thinking can be employed and can prove to be fruitful; we are not arguing that it is already a regular part of ecological methodology. It should also be clear that this fourdimensional approach to the allometries of metabolic ecology appears to be a productive line of research, although, admittedly, only time will tell whether the approach is ultimately correct. We thus have a case of analogical reasoning from physics allowing the formulation of new hypotheses in ecology, even though there are significant differences between the origin of the idea in physics and the application to scaling relationships in metabolic ecology. We also note that there is no suggestion of common cause here; indeed, it is not even clear that the relevant physics is in the business of providing causal explanations (Colyvan 2001:50-51). Rather, 
the analogy allows one to see abstract structural similarities, and this insight opens up interesting lines of ecological research. In our view, there do not need to be common causes or even similar causal networks in place in each case; abstract structural similarity alone is enough to generate the hypotheses in question. Testing of these hypotheses is then conducted in the usual ways and will, no doubt, prompt further work on understanding causal mechanisms, where appropriate.

\section{RATIO-DEPENDENT PREDATION VERSUS LAW OF MASS ACTION}

Models of species interaction have relied on analogies from physics ever since their original appearance nearly 100 years ago. For instance, Lotka's 1924 book was entitled Elements of Physical Biology but was reprinted in 1956 as Elements of Mathematical Biology (Lotka 1956) —an erroneous editorial change, in our opinion, as the original title explicitly emphasized the connections between ecology and physics. Volterra (1931), independently of Lotka, used the image of random encounters that was basic for physical chemistry, a subject in which Lotka majored in college. A more recent suggestion that complements the classical Lotka-Volterra model is based on a simple invariance or symmetry.

The notions of invariance and symmetry are central to a great deal of modern physics. Richard Feynman (1965) gives a typically clear account of invariancy of physical law with respect to translation in space, whereby only relative distances matter. This symmetry is not perfect, but it is a very useful approximation that holds almost everywhere.

Analogous to spatial translation (i.e., adding the same value to all spatial coordinates of a given physical system), there is a symmetry in the interaction of a consumer population with the resource it consumes. Interaction will be approximately constant if we multiply both the resource abundance and the consumer abundance by the same factor. Just as with physical invariancy, it does not hold perfectly and universally. For example, when consumers are rare, their growth will depend on the resource density itself and not on the per capita density, since their home ranges will not overlap. For a substantial range of values, however, the invariancy holds. This was noticed long ago when Contois (1959) grew E. coli in chemostat, and determined that the growth rate is a function of per capita, rather than absolute, sugar concentration.

The invariancy under discussion here has become known as ratio-dependent predation (Arditi and Ginzburg 1989) and has attracted considerable attention in ecology (reviewed by Abrams and Ginzburg 2000; Jensen and Ginzburg 2005), eventually entering the latest editions of standard ecology textbooks (Begon et al. 2006; Krebs 2008). Without going into the details of when this invariancy is or is not a good approximation, we simply draw attention to the analogy with translation invariance in physics. In fact, when viewed in logarithmic scale, as is the custom in ecology, the two principles are mathematically identical. We stress, however, that, in both cases, the invariances are only approximate and are violated in extreme situations.

Invariance is a central concept, and, for an adequate understanding of some phenomenon, we need to know when the phenomenon in question is changed and when it is not; that is, we need to know with respect to what is the phenomenon invariant. This we learned from physics long ago, and it also turns out to be useful in ecology, although the idea is put to different use in the ecological setting. Such analogical thinking opens up new possibilities that, in some cases, could not even be otherwise entertained. For example, exponential growth of both predator and prey is a possibility in the ratiodependent view, but not on the standard Lotka-Volterra model. Also, it is interesting to note that there are "balanced growth" models in economics that allow joint coordinated exponential expansion across several sectors (Cooley 1995). The core of the ratiodependent predation debate is whether Malthusian growth is truly fundamental in ecology or whether it disappears as species interact. 
Even though the Lotka-Volterra model is not invariant, when the consumer density is low, the physical image that the model suggests works better, as interference in consumption can be ignored. At the other extreme, when the density is high, interference is nearly perfect and ratio-dependent analogy works (Abrams and Ginzburg 2000). Both models turned out to use physical analogies-just different ones-and both have their domains of applicability. The existing tension is not about which one is right, but rather concerns the clear delineation of the domains of validity.

\section{INERTIA IN POPULATION GROWTH}

The suggestion that exponential growth (Malthus's law) in ecology is analogous to Newton's first law in physics is over 20 years old (Ginzburg 1986) and has since been adopted by others in ecology (e.g., Turchin 2001). Taking exponential growth to be the default state focuses attention on departures from unrestricted exponential growth and on the rate of change of population growth, rather than on rates of population growth. In more mathematical terms, this is to say that the population growth of a single species may be viewed as a two-dimensional process with another variable, in addition to abundance in joint simultaneous dynamics. It is important to note that the analogy with physics here is clear and is quite explicit in all the key publications on the topic (e.g., Ginzburg 1986; Colyvan and Ginzburg 2003a). Thinking about inertia in physics led to the inertial view of population dynamics via analogical reasoning, and it is a very natural chain of reasoning when viewed from a suitably abstract point of view (Ginzburg and Colyvan 2004).

One likely candidate for such a "hidden" variable is individual quality, and the mechanism of interaction is the so-called "maternal effect." The idea that the maternal effect can result in second-order dynamics of a single species is one that is at least 50 years old (Wellington 1957). In its quantitative form, it was developed in the 1990s and has since been summarized in Colyvan and Ginzburg (2003a), Ginzburg and Colyvan (2004), and also sympathetically discussed in Wagner (2005). The maternal effect as a mechanism for population cycles due to second-order internal dynamics remains a lively research area, with a large number of publications, including good experimental work (see review by Inchausti and Ginzburg 2009). Just as in the case of ratio dependence discussed above, the inertial idea of population dynamics is also finding its way into ecology textbooks (Begon et al. 2006), albeit as a minority and somewhat controversial view, but one worthy of investigation just the same. The dominant view is that the source of deviation from exponential growth lies in species interactions such as predator-prey relationships. It is our opinion that the relative emphasis on internal (inertial) versus external reasons for population dynamics may shift in the future towards a more pluralistic approach, where several mechanisms for departure from exponential growth are recognized. In any case, the analogy between exponential growth in ecology and uniform motion in physics has delivered an interesting new approach to population dynamics. The ultimate fate of this new approach is yet to be determined, but if it finds acceptance, the analogical reasoning in question will have led to genuine progress.

There are other examples of analogical thinking, both actual, such as Lotka's explicit use of analogies from the physics of chemical reactions to model population interactions (Kingsland 1985), and potential (i.e., other examples of where thinking in terms of symmetries might prove fruitful). But we trust we have made our point that analogical thinking from physics can and has been used to good effect in ecological theory building.

\section{ANAlogical Insights from Evolution}

In this section, we show that the kind of analogical reasoning we are advocating is a twoway street. We are not simply advancing a kind of physics envy, where ecologists should try to emulate the feats and methods of physics. We believe that physics can and has learned a great deal from biology, via similar analogical reasoning. An obvious and well-known example here 
is the groundbreaking work of Robert May (1974) on stability and bifurcations in population equations. This work was important in the development of chaos theory and opened the way for a better appreciation of complex systems, wherever they occur-ecology, physics, or elsewhere. Here we focus on a couple of less well-known examples where it appears that physicists have learned from one of the giants of biology: Charles Darwin.

\section{ANTHROPIC REASONING}

There is an interesting question in cosmology concerning the so-called "fine-tuning" of the universe. It turns out that many of the physical constants, such as the electron-proton mass ratio and the fine structure constant, could not have differed by more than a few percent from their actual values without resulting in a radically different universe. In particular, were some of these constants to be just slightly different, the universe would not contain carbon-based life and consciousness (Barrow and Tipler 1986, Cf. Weinberg 1993). It appears, therefore, that the universe is tailor-made for life. A variety of hypotheses have been advanced to account for this surprising fine-tuning of the universe, from religious intelligent design hypotheses to multiverse hypotheses. Let us consider the more serious scientific response: the multiverse hypothesis.

According to the multiverse response, there are many universes, either as a result of many big bangs or as a result of island universes arising from a single big bang. The details need not concern us. The main idea is that these universes are created with variation-perhaps random variation-of the many physical constants appearing in the laws of physics. We thus end up with infinitely many universes, and, in keeping with the fine-tuning observation, very few of them contain carbon-based life or consciousness. Under this hypothesis, there is no puzzle as to why there is some universe with the physical constants required for carbon-based life, just as there is no puzzle about why, after infinitely many card shuffles, there was one shuffle that left the cards arranged in descending suit order. The only puzzle left is why does our uni- verse contain life. The anthropic principle is now invoked. This principle is a selection principle that states that only universes with consciousness in them will have agents capable of wondering about their own and other universes. Again, there is no puzzle about why we find ourselves in one of the universes with consciousness, for we are conscious and universes without consciousness do not have anyone present in them to be puzzled. It is also not too much of a stretch to argue that universes with consciousness have to contain life of some sort, if not carbon-based life.

We are not endorsing or defending this line of response here (see Colyvan et al. 2005 for another line of response), but this is a serious scientific hypothesis, endorsed by quite a few physicists. Importantly, for present purposes, this response owes a great deal to Darwin. The fine-tuning argument, after all, is simply a reworked design argument, structurally the same as Paley's (1802) biology-based design argument. Where Paley appeals to the adaptation of organisms to their environments and praises the virtues of various human organs (such as the eye) for survival purposes, the new design argument appeals to the fine-tuning and presence of life itself in the universe. And the multiverse response combined with the anthropic principle is analogous to genetic variation and natural selection as a response to the original design argument. Focusing on the similarities between these two design arguments leads to an interesting Darwin-inspired response to the fine-tuning argument. A related response to fine-tuning is the fecund universe theory (Smolin 1997), according to which universes produce daughter universes and natural selection operates to favor those universes likely to have plentiful offspring. In the standard multiverse theory, the analogy with Darwinian evolutionary theory is clear enough, but, in fecund universe theory, the analogy carries over in almost every detail. Moreover, the Darwinian inspiration is quite explicit in the fecund universe theory.

\section{GRAVITATIONAL EXCLUSION}

The anthropic principle example just discussed is a direct application of a Darwinian 
evolution model in that there is both "mutation" when universes reproduce and "selection" via the anthropic principle, but our next example - the evolution of the solar system-is a little different. Planets, or the initial particles that formed them, do not reproduce, but they are certainly subject to a kind of "exclusion." Some of these particles joined the "planetesimals" and protoplanets, while others fell into the sun. In all cases, we view what we see today as a result of an historical process of elimination. This is a view present in all the theories of the 20th century, including the now discredited Chamberlin-Moutton theory-a biparental theory where planets grew from encounters between the sun and another star (Brush 1996). The prevailing current view is uniparental: all the initial material came from one source-a supernova-which provided the material for the sun, the planets, and the asteroids. This initial material is assumed to have been widely and randomly distributed, providing the celestial analogue of the biological random mutation. This material was then subjected to a kind of stability selection in that the particles in unstable regions of space would be drawn into the stable regions, thus increasing the number of particles in these stable regions. We therefore have a celestial analogue of differential selection. Recent discoveries of planets around distant stars, behaving very much like our own, is the single strongest argument towards the current view, even though there are other more direct arguments (Brush 1996). The current gradual and evolutionarylike theories are very different from the 19thcentury views of the meteoric origin of planets, which postulated that planets were originally meteors caught by the sun's gravitational field. The impact of Charles Darwin here is clear: he created an intellectual climate of evolutionary and selectionist thinking in biology and this, it would seem, was appropriated to good effect by astronomy.

A further example of this gravitational exclusion can be seen directly in the asteroid belt and the rings of Saturn. The rings of Saturn are relatively stable, but there are elliptical gaps representing unstable orbits. Any particle drifting into these regions will be dragged out again by the gravitational forces exerted by nearby bodies. Similarly, in the asteroid belt between Mars and Jupiter, there are well-known gaps-the Kirkwood gaps-that represent unstable orbits, selected against by the dynamics of the gravitational system of which they are a part (Arnol'd 1990). Again, this gravitational exclusion is similar to competitive exclusion in ecology. It might seem that this analogy is a little stretched. After all, as we have already pointed out, the gravitational exclusion case has no obvious analogue of reproduction. It has selection, and that selection is natural, as it is due to gravitation, but there is no "natural selection." Be that as it may, it is also clear that even partial analogies like this can be fruitful.

An interesting consequence of gravitational exclusion is that the unstable regions often place the surviving bodies at some distance from one another. This allows these bodies to be treated via the relatively simple Kepler's laws, which essentially approach the interactions of primary interest as two-body problems, thus enabling us to ignore other gravitational influences. We speculate that ecological allometries, the ecological analogue of Kepler's laws (Ginzburg and Colyvan 2004), may be explained by ecological elimination, similar to the way in which the simplicity arising from Kepler's laws was made possible by gravitational exclusion.

The message to be gleaned here is that it would appear as if physicists took natural selection seriously, and that it affected the way they thought about a variety of phenomena in the 150 years since Darwin published his theory of natural selection. Even if they didn't explicitly appeal to biological analogies, the intellectual climate after Darwin facilitated the making of such connections, and the connections are plain to see. The result has been several evolution-inspired advances in physics.

\section{Conclusion}

We have argued that we should not limit ourselves in where we search for new ideas and, in particular, that analogies drawn from other scientific disciplines are a legitimate and potentially fruitful means of generating these new ideas. Drawing attention to differences in kind between branches of science is 
counterproductive; electromagnetism learned from gravitation, just as evolution and economics have learned from each other. Instead, we should notice the points of contact and similarities between disciplines. No doubt, there are differences as well, but we should not let the differences blind us to the similarities and the lessons that we can learn from other branches of science. Indeed, as science progresses with more and more specialized sub-disciplines emerging, each with their own stock of tools and methods, the importance of one group of researchers learning from others via analogical thinking may become even more important.

The examples we presented are cases where analogical reasoning between ecology and physics has, at least potentially, enriched each discipline. Often this analogical reasoning is conducted by invoking the resources of mathematics, where abstraction away from detail helps in the appreciation of structural similarities (Colyvan 2001, 2002). At the very least, analogical thinking can give practitioners another means of generating interesting hypotheses, candidate laws, and theories, which, of course, need to be tested by the standards of the discipline in question. The suggestions in the third section of our paper need to be scrutinised and subjected to ecological confirmation or disconfirmation; the examples in our fourth section need to be subjected to the scrutiny of physicists.

There are undoubtedly dangers associated with analogical reasoning. Physics and ecology are different and they use different methods. Analogies between the two can be stretched too far, and, when this occurs, analogical thinking may well impede scientific progress. Illadvised analogies might invite the acceptance of assumptions warranted in one domain but not in another, and might even lead to faulty experimental designs (Mikkelson 1997). But our claim is not that analogical thinking will always prove to be fruitful-just that sometimes it can be. And we do not claim that there are no dead ends and pitfalls associated with analogical thinking; we simply believe that such thinking does not always lead to trouble. Indeed, the scientific enterprise itself offers no guarantees that any particular instance of a method will not mislead or lead to dead ends, so, in this regard, analogical thinking is no different from other tools available to scientists.

Newton once remarked that he had been fortunate enough to stand on the shoulders of giants. The giants he was referring to were probably Aristotle, Descartes, Galileo, Kepler, and others-all giants of physics, as well as giants of mathematics and philosophy in some cases. Ecologists and biologists have no hesitation in learning from the giants of their own respective disciplines: Lotka, Volterra, MacArthur, and of course Darwin. We have no quarrel with this; our suggestion is a modest one. We simply suggest that there is no need to limit ourselves to the giants of ecology and biology; rather, any giant will do-Newton, Darwin, Einstein, or Volterra. There are, no doubt, wondrous things to be seen from atop any of those shoulders.

\section{ACKNOWLEDGMENTS}

Thanks to Resit Akçakaya, Dan Dykhuizen, and the referees of this journal for many helpful suggestions. Mark Colyvan also wishes to acknowledge the Australian Centre of Excellence for Risk Analysis and the Commonwealth Environment Research Facilities Research Hub: Applied Environmental Decision Analysis for supporting his work on this paper.

\section{REFERENCES}

Abrams P. A., Ginzburg L. R. 2000. The nature of predation: prey dependent, ratio dependent or neither? Trends in Ecology and Evolution 15(8):337341.

Arditi R., Ginzburg L. R. 1989. Coupling in predator prey-dynamics: ratio-dependence. Journal of Theoretical Biology 139:311-326.

Arnol'd V. I. 1990. Huygens and Barrow, Newton an Hooke: Pioneers in Mathematical Analysis and Catas- trophe Theory from Evolvents to Quasicrystals. Basel (Switzerland): Birkhäuser.

Barrow J. D., Tipler F. J. 1986. The Anthropic Cosmological Principle. Oxford (UK): Oxford University Press.

Begon M., Townsend C. R., Harper J. L. 2006. Ecology: From Individuals to Ecosystems. Fourth Edition. Oxford (UK): Blackwell Publishing.

Berryman A. A. 2003. On principles, laws and theory in population ecology. Oikos 103(3):695-701. 
Blum J. J. 1977. On the geometry of four dimensions and the relationship between metabolism and body mass. Journal of Theoretical Biology 64:599601.

$\rightarrow$ Brown J. H., Gillooly J. F., Allen A. P., Savage V. M., West G. B. 2004. Toward a metabolic theory of ecology. Ecology 85:1771-1789.

Brush S. G. 1996. Fruitful Encounters: The Origin of the Solar System and of the Moon from Chamberlin to Apollo. Cambridge (UK): Cambridge University Press.

Colyvan M. 2001. The Indispensability of Mathematics. New York: Oxford University Press.

$\rightarrow$ Colyvan M. 2002. Mathematics and aesthetic considerations in science. Mind 111(441):69-74.

$\rightarrow$ Colyvan M., Garfield J. L., Priest G. 2005. Problems with the argument from fine tuning. Synthese 145(3): 325-338.

$\rightarrow$ Colyvan M., Ginzburg L. R. 2003a. The Galilean turn in population ecology. Biology and Philosophy 18(3):401414.

$\rightarrow$ Colyvan M., Ginzburg L. R. 2003b. Laws of nature anc laws of ecology. Oikos 101(3):649-653.

Contois D. E. 1959. Kinetics of bacterial growth: relationship between population density and specific growth rate of continuous cultures. Journal of Gen eral Microbiology 21:40-50.

Cooley T. F., editor. 1995. Frontiers of Business Cycle Research. Princeton (NJ): Princeton University Press.

Duhem P. 1954. The Aim and Structure of Physical The ory. Princeton (NJ): Princeton University Press.

Feynman R. 1965 The Character of Physical Law. London (UK): BBC.

$\rightarrow$ Ginzburg L. R. 1986. The theory of population dynamics: I. Back to first principles. Journal of The retical Biology 122:385-399.

Ginzburg L. R., Colyvan M. 2004. Ecological Orbits: How Planets Move and Populations Grow. New York: Oxford University Press.

$\rightarrow$ Ginzburg L. R., Damuth J. 2008. The space-lifetime hypothesis: viewing organisms in four dimensions literally. American Naturalist 171(1):125-131.

$\rightarrow$ Ginzburg L. R., Jensen C. X. J. 2004. Rules of thumb for judging ecological theories. Trends in Ecology and Evolution 19(3):121-126.

Hainsworth F. R. 1981. Animal Physiology: Adaptation in Function. Reading (MA): Addison-Wesley.

Hughes R. I. G. 1989. The Structure and Interpretation of Quantum Mechanics. Cambridge (MA): Harvard University Press.

Inchausti P., Ginzburg L. R. 2009. Maternal effects mechanism of population cycling: a formidable competitor to the traditional predator-prey view Philosophical Transactions of the Royal Society, Series $B$ 364:1117-1124.

$\rightarrow$ Jensen C. X. J., Ginzburg L. R. 2005. Paradoxes or theoretical failures? The jury is still out. Ecological Modelling 188(1):3-14
Kingsland S. E. 1985. Modelling Nature: Episodes in the History of Population Ecology. Chicago (IL): Chicago University Press.

Kleiber M. 1932. Body size and metabolism. Hilgardia 13:315-353.

Krebs C. J. 2008. Ecology: The Experimental Analysis of Distribution and Abundance. Sixth Edition. Upper Saddle River (NJ): Benjamin Cummings Press.

Lakatos I. 1970. Falsification and the methodology of scientific research programmes. Pages 91-195 in Criticism and the Growth of Knowledge, edited by I. Lakatos and A. Musgrave. Cambridge (UK): Cambridge University Press.

Lange M. 2005. Ecological laws: what would they be and why would they matter? Oikos 110(2):394403.

Lawton J. H. 1999. Are there general laws in ecology? Oikos 84:177-192.

Lockwood D. R. 2007. Ecology is not rocket science. Emergence: Complexity and Organization 9(2):49-58.

Lockwood D. R. 2008. When logic fails ecology. Quarterly Review of Biology 83(1):57-64.

Lotka A. J. 1956. Elements of Mathematical Biology. New York: Dover.

Lyon A., Colyvan M. 2008. The explanatory power of phase spaces. Philosophia Mathematica 16(2):227243.

May R. M. 1974. Biological populations with nonoverlapping generations: stable points, stable cycles, and chaos. Science 186(4164):645-647.

Mikkelson G. M. 1997. Methods and metaphors in community ecology: the problem of defining stability. Perspectives on Science 5(4):481-498.

O'Hara R. B. 2005. The anarchist's guide to ecological theory. Or, we don't need no stinkin' laws. Oikos 110(2):390-393.

Paley W. 1802. Natural Theology; or, Evidence of the Existence and Attributes of the Deity. London (UK): J. Faulder. [Reprint 2006. Oxford (UK): Oxford University Press.]

Quenette P. Y., Gerard J. F. 1993. Why biologists do not think like Newtonian physicists. Oikos 68:361363.

Quine W. V. O. 1980. Two dogmas of empiricism. Pages 20-46 in From a Logical Point of View, Second Revised Edition, by W. V. O. Quine. Cambridge (MA): Harvard University Press.

Rubner M. 1883. Über den einfluss der körpergrösse auf stoff-und kraftwechsel. Zeitchrift für Biologie 19: 535-562.

Smolin L. 1997. The Life of the Cosmos. Oxford (UK): Oxford University Press.

Turchin P. 2001. Does population ecology have general laws? Oikos 94:17-26.

Volterra V. 1931. Leçons sur la Théorie Mathématique de la Lutte pour la Vie. Pédigées par Marcel Brelot. Cahiers Scientifiques, Vol. 7. Paris (France): GauthierVillars. 
$\rightarrow$ Wagner G. 2005. Mothers driving cycles. Science 309 (5743):2001.

$\rightarrow$ Wellington W. G. 1957. Individual differences as a factor in population dynamics: the development of a problem. Canadian Journal of Zoology 35:293323.

Weinberg S. 1993. Dreams of a Final Theory: The Search for the Fundamental Laws of Nature. London (UK): Vintage.

Whitfield J. 2006. In the Beat of a Heart: Life, Energy, and the Unity of Nature. Washington (D.C.): Joseph Henry Press.

Associate Editor: Anurag Agrawal 